\title{
Falhas no uso de equipamentos de proteção individual pelos profissionais de saúde: revisão de literatura
}

\author{
Failures in the use of personal protective equipment by health professionals: literature \\ review
}
Fallas en el uso de equipos de protección personal por parte de profesionales de la salud: revisión de literatura

Thaiz Helena Lopes da Rocha ${ }^{1 *}$, Beatriz Ribas de Melo1, Icaro Santos Veras ${ }^{1}$, Juliana de Castro Vilanova1, Lucas Mainardo Rodrigues Bezerra1, Alba Angélica Nunes Mouta ${ }^{2}$, Camila Maila Fontinele Beltrão ${ }^{1}$, Ilvanete Tavares Beltrão ${ }^{1}$, Renata Paula Lima Beltrão ${ }^{1,2}$, Augusto César Beltrão da Silva ${ }^{1,2}$.

\section{RESUMO}

Objetivo: Revisar a literatura científica atual acerca de acidentes ocupacionais com material biológico, decorrentes da negligência no uso de equipamentos de proteção individual, associados a seus riscos e implicações socioeconômicas. Métodos: Trata-se de uma revisão bibliográfica de caráter qualitativo, construída a partir de artigos científicos publicados nos últimos cinco anos (2016-2020), apenas na língua portuguesa. Foram usados os bancos de dados: BVS, Scielo, LILACS e PubMed/MEDLINE. Resultados: Foram selecionados 38 artigos, em que 21 foram lidos integralmente e utilizados neste estudo. A análise viabilizou a percepção de que fatores como idade e gênero estão associados à ocorrência de acidentes com materiais biológicos, os quais oferecem risco à saúde do trabalhador por serem vetores de doenças infectocontagiosas, assim como causam impacto direto na economia, principalmente no âmbito da seguridade social. Considerações finais: A exposição dos profissionais a materiais biológicos, por meio de acidentes, feridas cirúrgicas contaminadas ou manipulação de pacientes com doenças infectocontagiosas, pode ser evitada com o uso adequado dos equipamentos de proteção individual, os quais constituem uma barreira eficaz contra possíveis ocorrências que possam comprometer a saúde do profissional.

Palavras-chave: Acidentes de trabalho, Exposição ocupacional, Equipamento de proteção individual, Agentes biológicos.

\section{ABSTRACT}

Objective: To review the current scientific literature on occupational accidents involving biological material, resulting from negligence in the use of personal protective equipment, associated with its risks and socioeconomic implications. Methods: This is a bibliographic review, based on scientific articles published in the last five years (2016-2020), only in Portuguese. The following databases were used: VHL, SciELO, LILACS and PubMed / MEDLINE. Results: 38 articles were selected, of which 21 were read in full and used in this study. The analysis made possible the perception that factors such as age and gender are associated with the occurrence of accidents with biological materials, which pose a risk to the health of the worker because they are vectors of contagious diseases, as well as causing a direct impact on the economy, mainly in the scope of social security. Final considerations: The exposure of professionals to biological materials, through accidents, contaminated surgical wounds or manipulation of patients with infectious diseases, can be avoided with the proper use of personal protective equipment, which constitutes an effective barrier against possible occurrences that may compromise the health of the professional.

Keywords: Works accidents, Occupational exposure, Personal protective equipment, Biological agents.

\section{RESUMEN}

Objetivo: Revisar la literatura científica actual sobre accidentes laborales que involucran material biológico, resultado de negligencia en el uso de equipo de protección personal, asociado con sus riesgos e implicaciones socioeconómicas. Métodos: Esta es una revisión bibliográfica, basada en artículos científicos publicados en los últimos cinco años (20162020), solo en portugués. Se utilizaron las siguientes bases de datos: VHL, Scielo, LILACS y PubMed / MEDLINE. Resultados: Se seleccionaron 38 artículos, de los cuales 21 fueron leídos en su totalidad y utilizados en este estudio. El análisis hizo posible la percepción de que factores como la edad y el género están asociados con la ocurrencia de accidentes con materiales biológicos, que representan un riesgo para la salud del trabajador porque son vectores de enfermedades infecciosas, además de causar un impacto directo en la economía, principalmente en el ámbito de la seguridad. Social Consideraciones finales: La exposición de los profesionales a materiales biológicos, a través de accidentes, heridas quirúrgicas contaminadas o la manipulación de pacientes con enfermedades infecciosas, se puede evitar con el uso adecuado de equipos de protección personal, que constituyen una barrera eficaz contra posibles eventos que pueden comprometer la salud del profesional.

Palabras-clave: Accidentes de trabajo, Exposición ocupacional, Equipo de protección personal, Agentes biológicos.

${ }^{1}$ Instituição de Educação Superior do Vale do Parnaíba (IESVAP), Parnaíba - PI. *E-mail: thaiz.lopes@hotmail.com

2 Universidade Federal do Delta do Parnaíba (UFDPar), Parnaíba - PI. 


\section{INTRODUÇÃO}

Historicamente, a classe dos profissionais de saúde não era considerada muito suscetível à ocorrência de acidentes de trabalho, mas, atualmente, esse cenário vem sofrendo alterações significativas. No ano de 2012 , o Brasil foi considerado o quarto país com maior índice de acidentes laborais, custando cerca de U\$\$7 bilhões. Ressalta-se que em profissionais de saúde esses riscos são cada vez mais proeminentes, devido ao contato contínuo com agentes biológicos. Tal realidade está relacionada a práticas indevidas no uso de equipamentos de proteção individual (EPI) e equipamentos de proteção coletiva (EPC), bem como à subutilização de estratégias de proteção contra agentes contaminantes, como indisponibilidade dos equipamentos de proteção, fiscalização e instrução ineficazes, descarte inadequado de perfurocortantes, entre outras insalubridades encontradas no serviço de saúde (ARANTES MC, et al., 2017).

O termo biossegurança se originou nos anos 70 nos Estados Unidos, mas apenas nos anos 80 foram efetivamente criados manuais relativos ao tema, em que se destaca o criado pelo Centers for Disease Control and Prevention (CDC), o qual discorreu acerca de medidas preventivas contra agentes biológicos no ambiente de trabalho. O íntegro conhecimento e compreensão dos princípios de biossegurança são de extrema importância, sendo indispensáveis para a plenitude de sua execução (SALLES LL, et al., 2019).

Com intuito de promover a qualidade salutar de profissionais ligados à área da saúde, foi aprovada a norma regulamentadora 32 (NR32), de extrema importância para trabalhadores do serviço de saúde e que possui a finalidade de definir as diretrizes básicas para implementação de medidas de garantia da segurança e da saúde desses profissionais. Embora a norma seja elaborada especificamente no âmbito dos profissionais de saúde, ainda há inserção de em outras NRs que também os favorecem, ainda que não sejam especificas para este desígnio (LIMA RJV, et al. 2017).

Segundo Simão SAF, et al. (2017), ainda há a necessidade de difundir o conhecimento sobre a normativa, visto que o trabalhador deve ser um sujeito ativo no processo de trabalho/saúde, capaz de compreender o impacto do trabalho sobre a saúde e, dessa forma, intervir politicamente para transformar a sua realidade. Segundo Norma Regulamentadora 6 (NR 6) é dever do empregador fornecer todo e qualquer paramento para a segurança do trabalhador os EPIs e EPC, além de promover a capacitação com informativos de fácil compreensão; em caso de acidentes, medidas devem ser tomadas imediatamente, visando atingir melhores condições de trabalho (SOARES RZ, et al., 2018).

A elaboração de protocolos de atuação após exposições a materiais biológicos descreve procedimentos primordiais a áreas expostas, aconselhamento ao acidentado, avaliação do acidente (tipo de contato, fonte do contato) e notificação do mesmo. Entre os anos de 2007 e 2010 houve um aumento de $108 \%$ de casos de acidentes com agentes biológicos notificados. Presume-se que essas ocorrências sejam subestimadas, pois muitas vezes o medo do desemprego, a culpabilidade, a falta de organização do atendimento necessário ao trabalhador e, ainda, a descrença sobre acidentes dessa natureza impedem a devida notificação de tais incidentes (CORDEIRO TMSC, et al. 2016).

Profissionais da saúde são diretamente ligados a riscos infecciosos, visto que requerem proximidade física com pacientes além de risco com o manuseio de materiais infectados. Segundo a Organização Internacional do Trabalho (OIT) são 317 milhões de acidentes de trabalho (AT) em todo mundo e cerca de 160 milhões possuem prejuízos relacionados à saúde. No ano de 2007 cerca de 15,85\% dos 653.097 casos de AT são relacionados a trabalhadores da área de saúde.

A pequena atenção dada a esse questionamento é preocupante visto isso é necessário à conscientização de profissionais de saúde. $O$ ambulatório não é mais apenas o único ambiente de risco a estes profissionais após a criação Estratégia Saúde da Família (ESF), que realizam visitas domiciliares, logo possuem maior contato com pacientes que possam estar contaminados com algum patógeno (TELES AS, et al., 2016).

Desse modo, presente estudo teve como objetivo discorrer, a partir de uma revisão bibliográfica, sobre as falhas no uso de equipamentos de proteção individual (EPIs) pelos profissionais de saúde. Na abordagem, optou-se por especificar os riscos da exposição ocupacional, os grupos mais suscetíveis e os efeitos socioeconômicos envolvidos. 


\section{MÉTODOS}

Este artigo trata-se de uma revisão bibliográfica cuja abordagem aproxima-se da qualitativa e apresenta o propósito de gerar, de forma ordenada, uma união de estudos para a avaliação do tema proposto. As fases empregadas foram: análise de diferentes estudos, seleção de artigos específicos, determinação das informações selecionadas, revisão do estudo.

Os critérios de inclusão utilizados foram: 21 artigos nacionais ou internacionais publicados em português ou inglês; estudos dos últimos cinco anos, de janeiro de 2016 até janeiro de 2020, que estivessem disponíveis integralmente online nas bases pesquisadas e que tivessem como tema central acidentes ocupacionais com material biológico. Foram excluídos 17 artigos anteriores à data estabelecida, que não se encaixavam na temática apresentada e/ou que não abordavam os riscos de profissionais de saúde.

A respeito do idioma, a totalidade dos artigos encontrava-se na língua portuguesa. As bases de dados utilizadas foram: Biblioteca Virtual em Saúde (BVS), Literatura Latino-Americana e do Caribe em Ciências da Saúde (LILACS), Scientific Eletronic Library Online (SciELO) e Medical Literature Analysis and Retrieval System Online (MEDLINE) via National Library of Medicine (NLM/PubMed), com consulta aos Descritores em Ciência da Saúde (DeCS): Equipamento de Proteção Individual; Acidente de Trabalho; Fatores Biológicos; Pessoal de Saúde; Exposição Ocupacional.

A finalização deu-se a partir da discussão entre os autores e determinação ideias convergentes, o que permitiu a concretização das discussões por meio de uma análise que segue os princípios de autoria dos trabalhos científicos.

Figura 1 - Critérios de inclusão e exclusão

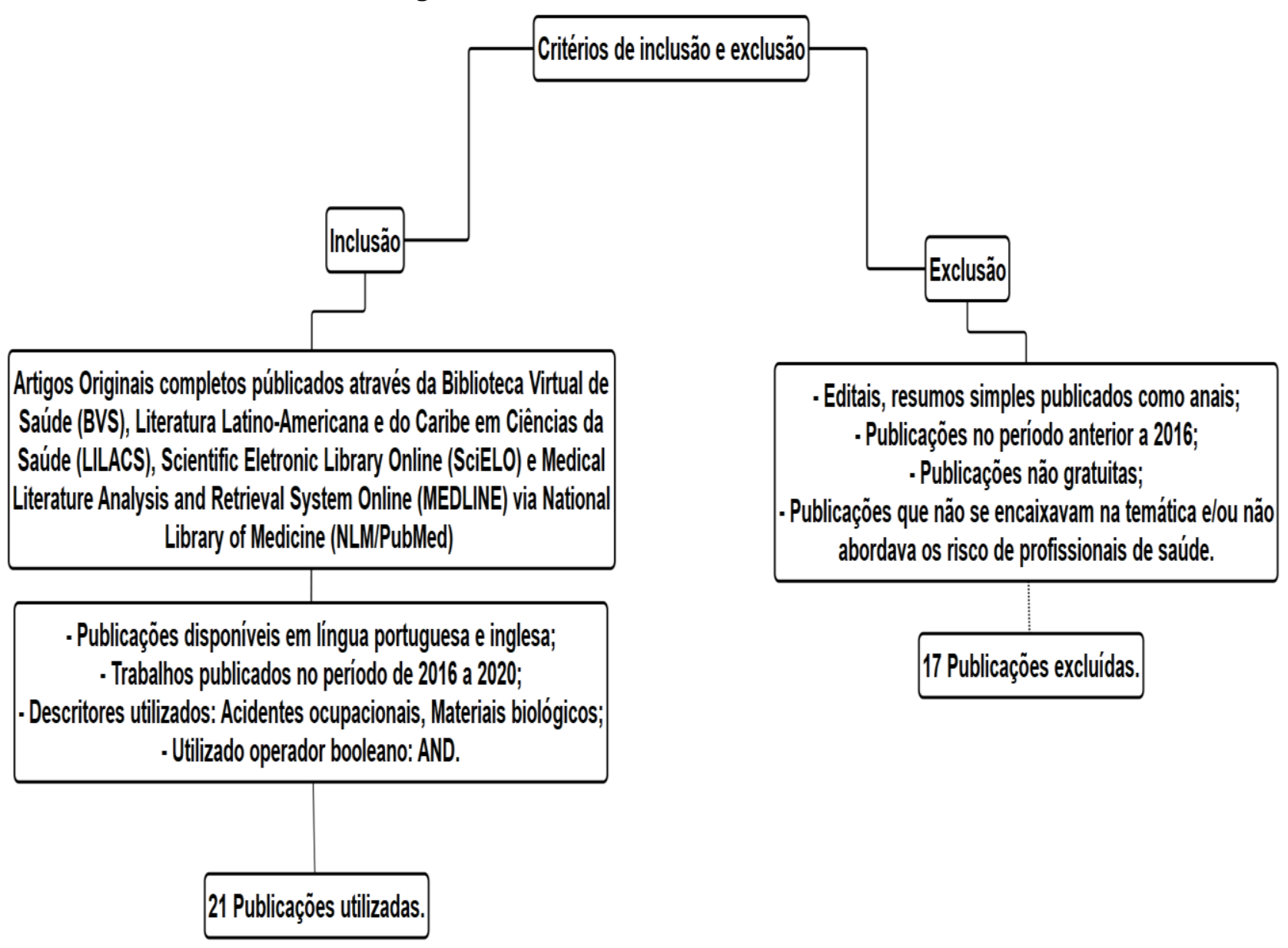

Fonte: Rocha THL, et al., 2020. 


\section{Revista Eletrônica Acervo Saúde / Electronic Journal Collection Health | ISSN 2178-2091}

Quadro 1 - Distribuição das obras utilizadas de acordo com autores, ano, título, periódico, objetivos e resultados do estudo.

\begin{tabular}{|c|c|c|c|c|}
\hline Autores e ano & Título & Revista & Objetivo de estudo & Resultados \\
\hline \multirow[b]{2}{*}{$\begin{array}{l}\text { ARANTES MC, } \\
\text { et al., (2017) }\end{array}$} & $\begin{array}{l}\text { Acidentes com material biológico em } \\
\text { trabalhadores de serviços de saúde }\end{array}$ & \multirow[b]{2}{*}{$\begin{array}{l}\text { Cogitare } \\
\text { Enfermagem }\end{array}$} & \multirow[b]{2}{*}{$\begin{array}{l}\text { Caracterizar os acidentes de trabalho com material } \\
\text { biológico em trabalhadores de um serviço de saúde. }\end{array}$} & \multirow{2}{*}{$\begin{array}{l}\text { Dentre os } 1.061 \text { acidentes de trabalho com material biológico, } \\
58,1 \% \text { ocorreram com auxiliares/ técnicos de enfermagem } \\
\text { ( } 82,7 \% \text { do sexo feminino). } 88,2 \% \text { ocorreram por meio de } \\
\text { exposição percutânea, } 66,1 \% \text { pela utilização de agulhas com } \\
\text { lúmen e } 21,9 \% \text { decorrentes do descarte inadequado de } \\
\text { material perfurocortante. }\end{array}$} \\
\hline & $\begin{array}{l}\text { Occupational accidents with biological } \\
\text { material among healthcare workers }\end{array}$ & & & \\
\hline \multirow{2}{*}{$\begin{array}{l}\text { BALTHAZAR } \\
\text { MAP, et al., } \\
\text { (2017) }\end{array}$} & $\begin{array}{l}\text { Gestão dos riscos ocupacionais nos } \\
\text { serviços hospitalares: uma análise reflexiva }\end{array}$ & \multirow{2}{*}{$\begin{array}{l}\text { Revista de } \\
\text { Enfermagem } \\
\text { UFPE }\end{array}$} & \multirow{2}{*}{$\begin{array}{l}\text { Refletir sobre os riscos ocupacionais aplicados à } \\
\text { gestão de segurança no ambiente hospitalar }\end{array}$} & \multirow{2}{*}{$\begin{array}{l}\text { A segurança e saúde no trabalho estão determinadas por meio } \\
\text { das Normas Regulamentadoras do Ministério do Trabalho e } \\
\text { Emprego. O controle dos agentes de risco pode ser realizado } \\
\text { por meio de ações mitigadoras como o uso de EPIs } \\
\text { específicos. }\end{array}$} \\
\hline & $\begin{array}{l}\text { Occupational risk management in hospital } \\
\text { services: a reflective analysis }\end{array}$ & & & \\
\hline \multirow{2}{*}{$\begin{array}{l}\text { BATISTA JR, et } \\
\text { al., (2017) }\end{array}$} & $\begin{array}{l}\text { Conhecimento da equipe de enfermagem } \\
\text { perante os principais tipos de infecções } \\
\text { hospitalares }\end{array}$ & \multirow{2}{*}{$\begin{array}{l}\text { Revista de } \\
\text { Enfermagem } \\
\text { UFPE }\end{array}$} & \multirow{2}{*}{$\begin{array}{l}\text { Identificar o conhecimento da equipe de } \\
\text { enfermagem acerca das infecções hospitalares }\end{array}$} & \multirow{2}{*}{$\begin{array}{l}100 \% \text { dos enfermeiros e } 96,7 \% \text { dos técnicos de enfermagem } \\
\text { têm conhecimento sobre infecção hospitalar; embora apenas } \\
80 \% \text { dos enfermeiros e } 70 \% \text { dos técnicos de enfermagem } \\
\text { usem o EPIs corretamente. }\end{array}$} \\
\hline & $\begin{array}{l}\text { Knowledge of the nursing team in the main } \\
\text { types of hospital infections }\end{array}$ & & & \\
\hline \multirow{2}{*}{$\begin{array}{l}\text { BOEIRA ER, et } \\
\text { al., (2019) }\end{array}$} & $\begin{array}{l}\text { Controle de infecções e medidas de } \\
\text { segurança do paciente abordados em } \\
\text { projetos pedagógicos da enfermagem }\end{array}$ & \multirow{2}{*}{$\begin{array}{l}\text { Revista da } \\
\text { Escola de } \\
\text { Enfermagem da } \\
\text { USP }\end{array}$} & \multirow{2}{*}{$\begin{array}{l}\text { Caracterizar o ensino acerca da segurança do } \\
\text { paciente e das medidas de precauções-padrão para } \\
\text { prevenção e controle de infecções, nos cursos de } \\
\text { graduação em enfermagem. }\end{array}$} & \multirow{2}{*}{$\begin{array}{l}\text { Trinta e nove disciplinas comtemplaram o ensino das medidas } \\
\text { de prevenção e controle das infecções, e os temas mais } \\
\text { abordados foram equipamento de proteção individual e higiene } \\
\text { das mãos. }\end{array}$} \\
\hline & $\begin{array}{l}\text { Infection control and patient safety } \\
\text { measures addressed in nursing } \\
\text { pedagogical projects }\end{array}$ & & & \\
\hline \multirow{2}{*}{$\begin{array}{l}\text { CARVALHO DC, } \\
\text { et al., (2018) }\end{array}$} & $\begin{array}{l}\text { Acidentes de trabalho com material } \\
\text { biológico na equipe de enfermagem de um } \\
\text { hospital do Centro-Oeste brasileiro }\end{array}$ & \multirow{2}{*}{$\begin{array}{l}\text { Escola Anna } \\
\text { Nery Revista de } \\
\text { Enfermagem }\end{array}$} & \multirow{2}{*}{$\begin{array}{l}\text { Investigar e descrever a ocorrência e as } \\
\text { características dos Acidentes de Trabalho (AT) com } \\
\text { Material Biológico Potencialmente Contaminado } \\
\text { (MBPC), a frequência e as variáveis relacionadas à } \\
\text { ocorrência entre as categorias de enfermagem. }\end{array}$} & \multirow{2}{*}{$\begin{array}{l}\text { Um total de } 46,6 \% \text { dos profissionais relatou já ter sofrido } \\
\text { exposição à MBPC, e } 60,9 \% \text { referiram não ter realizado a } \\
\text { notificação e } 42,6 \% \text { afirmaram terem participado de } \\
\text { treinamentos sobre a temática. Os auxiliares foram mais } \\
\text { acometidos, quando comparados a enfermeiros. }\end{array}$} \\
\hline & $\begin{array}{l}\text { Work incidents with biological material in } \\
\text { the nursing team of a } \\
\text { hospital in Mid-Western Brazil }\end{array}$ & & & \\
\hline \multirow{2}{*}{$\begin{array}{l}\text { CARVALHO } \\
\text { HEF, et al., } \\
\text { (2019) }\end{array}$} & $\begin{array}{l}\text { Visão dos profissionais de enfermagem } \\
\text { quanto aos riscos ocupacionais e acidentes } \\
\text { de trabalho na central de material e } \\
\text { esterilização }\end{array}$ & \multirow{2}{*}{$\begin{array}{l}\text { Revista de } \\
\text { Pesquisa } \\
\text { (Universidade } \\
\text { Federal do } \\
\text { Estado do Rio } \\
\text { de Janeiro) }\end{array}$} & \multirow{2}{*}{$\begin{array}{l}\text { Explorar a visão dos profissionais de enfermagem } \\
\text { quanto aos riscos ocupacionais e acidentes } \\
\text { ocupacionais na Central de Material Esterilização }\end{array}$} & \multirow{2}{*}{$\begin{array}{l}\text { Emergiram três temas após as entrevistas: Riscos presentes } \\
\text { no ambiente de trabalho; A visão da Equipe de Enfermagem } \\
\text { sobre os acidentes na CME; e Assistência prestada aos } \\
\text { profissionais acidentados. }\end{array}$} \\
\hline & $\begin{array}{l}\text { Nursing professionals' perspective on } \\
\text { occupational risks and work accidents in the } \\
\text { sterilization and materials processing center }\end{array}$ & & & \\
\hline \multirow{2}{*}{$\begin{array}{l}\text { CORDEIRO } \\
\text { TMSC, et al., } \\
\text { (2016) }\end{array}$} & $\begin{array}{l}\text { Acidentes de trabalho com exposição à } \\
\text { material biológico: Descrição dos casos na } \\
\text { Bahia }\end{array}$ & \multirow{2}{*}{$\begin{array}{l}\text { Revista de } \\
\text { Epidemiologia e } \\
\text { Controle de } \\
\text { Infecção }\end{array}$} & \multirow{2}{*}{$\begin{array}{l}\text { Descrever os fatores de risco relacionados a } \\
\text { acidentes de trabalho com exposição a material } \\
\text { biológico e as condutas adotadas pós-exposição } \\
\text { dos casos notificados no Sistema de Informação de } \\
\text { Agravos de Notificação (SINAN) no estado da Bahia } \\
\text { em } 2012\end{array}$} & \multirow{2}{*}{$\begin{array}{l}\text { Maior ocorrência dos acidentes ocupacionais com exposição a } \\
\text { materiais biológicos na } \\
\text { Bahia na população feminina }(78,1 \%) \text { e na faixa etária entre } \\
30-49 \text { anos }(51,5 \%) \text {; o sangue foi o fluido de maior contato nos } \\
\text { acidentes com } 75,2 \% \text {, por meio da via percutânea }(71,5 \%) \text {. }\end{array}$} \\
\hline & $\begin{array}{l}\text { Occupational accidents with exposure to } \\
\text { biological material: } \\
\text { Description of cases in Bahia }\end{array}$ & & & \\
\hline
\end{tabular}




\section{Revista Eletrônica Acervo Saúde / Electronic Journal Collection Health | ISSN 2178-2091}

\begin{tabular}{|c|c|c|c|c|}
\hline Autores e ano & Título & Revista & Objetivo de estudo & Resultados \\
\hline \multirow{2}{*}{$\begin{array}{l}\text { CUNHA NA, } \\
(2017)\end{array}$} & $\begin{array}{l}\text { Acidentes de trabalho com exposição a } \\
\text { material biológico: análise epidemiológica e } \\
\text { percepção das vítimas, em Uberlândia - } \\
\text { MG }\end{array}$ & \multirow{2}{*}{$\begin{array}{l}\text { Sistema de } \\
\text { Bibliotecas da } \\
\text { UFU, MG, } \\
\text { Brasil. }\end{array}$} & \multirow{2}{*}{$\begin{array}{l}\text { Traçar o perfil dos profissionais envolvidos, } \\
\text { identificar as circunstâncias dos acidentes e } \\
\text { compreender a } \\
\text { perspectiva dos trabalhadores quanto às causas e } \\
\text { meios de prevenção do ATMB. }\end{array}$} & \multirow{2}{*}{$\begin{array}{l}\text { Acidentes com materiais biológicos tiveram maior prevalência } \\
\text { entre os técnicos de enfermagem, do gênero feminino, sendo } \\
\text { os instrumentos perfurantes os principais agentes materiais } \\
\text { envolvidos e o sangue a principal substância orgânica } \\
\text { relacionada às ocorrências. }\end{array}$} \\
\hline & $\begin{array}{l}\text { Work accidents with exposure to biological } \\
\text { material: epidemiological analysis and } \\
\text { perception of victims, in Uberlândia - MG }\end{array}$ & & & \\
\hline \multirow{2}{*}{$\begin{array}{l}\text { FERNANDES } \\
\text { MA, et al., (2017) }\end{array}$} & $\begin{array}{l}\text { Utilização de equipamentos de proteção } \\
\text { individual: interfaces com o conhecimento } \\
\text { dos profissionais de saúde. }\end{array}$ & \multirow{2}{*}{$\begin{array}{l}\text { Revista } \\
\text { Prevenção de } \\
\text { Infecção e } \\
\text { Saúde. }\end{array}$} & \multirow{2}{*}{$\begin{array}{l}\text { Analisar o conhecimento de profissionais de saúde } \\
\text { de um Ambulatório } \\
\text { Escola sobre o uso de Equipamentos de Proteção } \\
\text { Individual (EPI) e sua relação com a adesão aos } \\
\text { mesmos. }\end{array}$} & \multirow{2}{*}{$\begin{array}{l}\text { A partir dos depoimentos emergiram três categorias } \\
\text { semânticas: "Equipamentos de Proteção Individual: uma } \\
\text { maneira mais segura de trabalho" a amostra com } 229 \\
\text { enfermeiros demonstrou alta incidência de riscos biológicos. } \\
\text { "Utilização dos EPIs: uma responsabilidade coletiva" e } \\
\text { "Utilização seletiva dos EPIs". }\end{array}$} \\
\hline & $\begin{array}{l}\text { Use of personal protective equipment: } \\
\text { interfaces with knowledge of health } \\
\text { professionals }\end{array}$ & & & \\
\hline \multirow{2}{*}{$\begin{array}{l}\text { GOMES SCS, } \\
\text { CALDAS AJM. } \\
\text { (2019) }\end{array}$} & $\begin{array}{l}\text { Incidência de acidentes de trabalho com } \\
\text { exposição a material biológico em } \\
\text { profissionais de saúde no Brasil, 2010- } \\
2016 \text {. }\end{array}$ & \multirow{2}{*}{$\begin{array}{l}\text { Revista } \\
\text { Brasileira de } \\
\text { Medicina do } \\
\text { Trabalho. }\end{array}$} & \multirow{2}{*}{$\begin{array}{l}\text { Descrever as características e as condutas } \\
\text { adotadas pós-exposição entre os profissionais de } \\
\text { saúde vítimas de Acidente de trabalho com } \\
\text { exposição a material biológico no Brasil. }\end{array}$} & \multirow{2}{*}{$\begin{array}{l}\text { Entre os casos notificados, prevaleceu a população feminina, } \\
\text { na faixa etária entre } 25 \neg 31 \text { anos, com } 12 \text { anos ou mais de } \\
\text { escolaridade, e auxiliares e técnicos de enfermagem ( } 64,71 \%) \text {. } \\
\text { O sangue foi o material biológico mais envolvido nos acidentes } \\
\text { (74,93\%) por meio da via percutânea }(75,33 \%) \text {, com a agulha } \\
\text { sendo o principal agente causador ( } 57,59 \%) \text {, e } 29,71 \% \text { dos } \\
\text { pesquisados não utilizavam luvas no momento do acidente. }\end{array}$} \\
\hline & $\begin{array}{l}\text { Incidence of work accidents involving } \\
\text { exposure to biological materials among } \\
\text { healthcare workers in Brazil, 2010-2016. }\end{array}$ & & & \\
\hline \multirow{2}{*}{$\begin{array}{l}\text { LA-ROTTA EIG, } \\
\text { et al. (2020) }\end{array}$} & $\begin{array}{l}\text { Conhecimento e adesão como fatores } \\
\text { associados a acidentes com agulhas } \\
\text { contaminadas com material biológico: Brasil } \\
\text { e Colômbia. }\end{array}$ & \multirow{2}{*}{$\begin{array}{l}\text { Ciência \& } \\
\text { Saúde Coletiva }\end{array}$} & \multirow{2}{*}{$\begin{array}{l}\text { Avaliar a prevalência de acidentes com material } \\
\text { biológico (AT-MB), o nível de adesão às Precauções } \\
\text { Padrão (PP) e o conhecimento sobre patógenos } \\
\text { transmissíveis pelo sangue e fatores associados } \\
\text { entre trabalhadores e estudantes da saúde, no } \\
\text { marco da implementação da norma NR-32. }\end{array}$} & \multirow{2}{*}{$\begin{array}{l}\text { Foram avaliados } 965 \text { indivíduos (348 estudantes e } 617 \\
\text { profissionais). O conhecimento teve média de } 10,98 \text { com } \\
\text { mediana de } 11(10,12) \text { e } \alpha-C r \text { de } 0,625 \text {. A média de adesão } \\
\text { foi de } 30,74 \text { com mediana de } 31(28,34) \text { e a-Cr de } 0,745, \\
\text { associando-se a País, grupo (estudantes) e percepção de } \\
\text { risco. Entre os fatores associadas ao relato de AT-MB } \\
\text { encontraram-se o conhecimento, a adesão às PP, País de } \\
\text { origem e ter tomado o esquema completo de vacinação contra } \\
\text { Hepatites B. }\end{array}$} \\
\hline & $\begin{array}{l}\text { Knowledge and compliance as factors } \\
\text { associated with accidents with needles } \\
\text { contaminated with biological material: Brazil } \\
\text { and Colombia }\end{array}$ & & & \\
\hline \multirow[t]{2}{*}{$\begin{array}{l}\text { LIMA RJV, et al., } \\
(2017\end{array}$} & $\begin{array}{l}\text { Agentes biológicos e equipamentos de } \\
\text { proteção individual e coletiva: } \\
\text { conhecimento e utilização entre } \\
\text { profissionais. }\end{array}$ & \multirow{2}{*}{$\begin{array}{l}\text { Revista } \\
\text { Prevenção de } \\
\text { Infecção e } \\
\text { Saúde }\end{array}$} & \multirow{2}{*}{$\begin{array}{l}\text { Descrever o conhecimento do conceito de agentes } \\
\text { biológicos e equipamentos de proteção por } \\
\text { profissionais de saúde em ambiente hospitalar, bem } \\
\text { como a utilização dos equipamentos de proteção } \\
\text { individual e coletiva. }\end{array}$} & \multirow{2}{*}{$\begin{array}{l}84,5 \%, 95,4 \% \text { e } 79,2 \% \text { dos profissionais de saúde } \\
\text { autodeclararam o conhecimento dos conceitos de agentes } \\
\text { biológicos, equipamentos de proteção individual e coletiva, } \\
\text { respectivamente. No que diz respeito à utilização dos } \\
\text { equipamentos de proteção individual, os mais citados foram } \\
\text { luvas }(32,1 \%), \text { jaleco }(29,2 \%) \text { e máscara }(28,1 \%) \text {. Já os de } \\
\text { proteção coletiva, a caixa de perfurocortante }(63,7 \%) \text { foi a mais } \\
\text { citada, seguida das pias de lavagem }(58,6 \%) \text { e autoclaves } \\
(20,8 \%) \text {. }\end{array}$} \\
\hline & $\begin{array}{l}\text { Biological agents and personal and } \\
\text { collective protection equipment: knowledge } \\
\text { and use among professional. }\end{array}$ & & & \\
\hline \multirow[b]{2}{*}{$\begin{array}{l}\text { MIRANDA FMD, } \\
\text { et al., (2017) }\end{array}$} & $\begin{array}{l}\text { Perfil dos trabalhadores brasileiros vítimas } \\
\text { de acidente de trabalho com fluidos } \\
\text { biológicos. }\end{array}$ & \multirow[b]{2}{*}{$\begin{array}{l}\text { Revista } \\
\text { Brasileira de } \\
\text { Enfermagem }\end{array}$} & \multirow[b]{2}{*}{$\begin{array}{l}\text { Caracterizar os trabalhadores brasileiros vítimas de } \\
\text { acidente de trabalho com fluidos biológicos }\end{array}$} & \multirow{2}{*}{$\begin{array}{l}\text { A maior densidade de incidência ocorreu no sexo feminino } \\
\text { com } 0,8 \text { caso a cada } 1.000 \text { trabalhadores/ano ( } \mathrm{n}=222.042 ; \\
77,9 \%) ; \text { na faixa etária de } 20 \text { a } 24 \text { anos com } 0,6 \text { caso a cada } \\
1.000 \text { trabalhadores/ano }(\mathrm{n}=64.221 ; 23,3 \% \text { ); com Ensino } \\
\text { Médio e Superior incompleto com } 0,6 \text { caso a cada } 1.000 \\
\text { trabalhadores/ano }(\mathrm{n}=141.275 ; 49,6 \% \text { ). Verificou-se que } \\
\text { esses acidentes ocorreram entre } 23 \text { subgrupos ocupacionais. } \\
\text { Em sua maioria, os trabalhadores possuíam vínculo } \\
\text { empregatício e tiveram emitida a Comunicação de Acidente de } \\
\text { Trabalho. }\end{array}$} \\
\hline & $\begin{array}{l}\text { Profile of Brazilian workers victims of } \\
\text { occupational accidents with biological fluids }\end{array}$ & & & \\
\hline
\end{tabular}




\section{Revista Eletrônica Acervo Saúde / Electronic Journal Collection Health | ISSN 2178-2091}

\begin{tabular}{|c|c|c|c|c|}
\hline Autores e ano & Título & Revista & Objetivo de estudo & Resultados \\
\hline \multirow{2}{*}{$\begin{array}{l}\text { NEGRINHO } \\
\text { NBS, et al., } \\
\text { (2017) }\end{array}$} & $\begin{array}{l}\text { Fatores associados à exposição } \\
\text { ocupacional com material biológico entre } \\
\text { profissionais de enfermagem }\end{array}$ & \multirow{2}{*}{$\begin{array}{l}\text { Revista } \\
\text { Brasileira de } \\
\text { Enfermagem }\end{array}$} & \multirow{2}{*}{$\begin{array}{l}\text { Identificar os fatores associados à exposição } \\
\text { ocupacional com material biológico entre } \\
\text { profissionais de enfermagem. }\end{array}$} & \multirow{2}{*}{$\begin{array}{l}\text { Dentre } 226 \text { profissionais entrevistados, } 17,3 \% \text { sofreram } \\
\text { exposição ocupacional com material biológico potencialmente } \\
\text { contaminado, sendo } 61,5 \% \text { por via percutânea. Fatores, como } \\
\text { a faixa etária }(p=0,003), \text { a experiência na enfermagem } \\
(p=0,015) \text { e na instituição, }(p=0,032) \text { estiveram associados ao } \\
\text { acidente. }\end{array}$} \\
\hline & $\begin{array}{l}\text { Factors associated with occupational } \\
\text { exposure to biological material among } \\
\text { nursing professional }\end{array}$ & & & \\
\hline \multirow{2}{*}{$\begin{array}{l}\text { SALLES LL, } \\
\text { ANJOS JM. } \\
\text { (2019) }\end{array}$} & $\begin{array}{l}\text { Equipamentos de proteção individual no } \\
\text { contexto laboral da enfermagem }\end{array}$ & \multirow[b]{2}{*}{$\begin{array}{l}\text { Revista } \\
\text { UNINGÁ }\end{array}$} & \multirow{2}{*}{$\begin{array}{l}\text { Identificar através da literatura os equipamentos de } \\
\text { proteção individual no contexto laboral da } \\
\text { enfermagem e a necessidade de utilização dos } \\
\text { mesmos. }\end{array}$} & \multirow{2}{*}{$\begin{array}{l}\text { Levantado o problema das altas sobrecargas de trabalho, o } \\
\text { dimensionamento inadequado do quadro de profissionais e a } \\
\text { carência de EPIs em quantidade e qualidade; por outro lado, } \\
\text { os profissionais que pouco dão importância a sua segurança } \\
\text { no local de trabalho, resultando na percepção que esses } \\
\text { profissionais têm do uso dos EPl's. }\end{array}$} \\
\hline & $\begin{array}{l}\text { Individual protective equipment in the work } \\
\text { contexto of nursing }\end{array}$ & & & \\
\hline \multirow{2}{*}{$\begin{array}{l}\text { SARDEIRO TL, } \\
\text { et al. (2019) }\end{array}$} & $\begin{array}{l}\text { Acidente de trabalho com material } \\
\text { biológico: fatores associados ao abandono } \\
\text { do acompanhamento clínico-laboratorial }\end{array}$ & \multirow{2}{*}{$\begin{array}{l}\text { Revista da } \\
\text { Escola de } \\
\text { Enfermagem da } \\
\text { USP }\end{array}$} & \multirow{2}{*}{$\begin{array}{l}\text { Analisar a epidemiologia do abandono do } \\
\text { acompanhamento clínico e laboratorial entre } \\
\text { trabalhadores da saúde que sofreram acidentes com } \\
\text { material biológico }\end{array}$} & \multirow{2}{*}{$\begin{array}{l}\text { A maioria das exposições analisadas envolvem mulheres com } \\
\text { ensino médio completo e pertencentes à equipe de } \\
\text { enfermagem. Os acidentes ocorreram predominantemente por } \\
\text { lesão percutânea envolvendo agulha com lúmen durante a } \\
\text { administração de medicamentos ou acesso vascular. A taxa de } \\
\text { abandono de acompanhamento foi } 41,5 \% \text {. }\end{array}$} \\
\hline & $\begin{array}{l}\text { Work accidents with biological material: } \\
\text { factors associated with abandoning clinical } \\
\text { and laboratory follow-up }\end{array}$ & & & \\
\hline \multirow[b]{2}{*}{$\begin{array}{l}\text { SOARES RZ, et } \\
\text { al. (2019) }\end{array}$} & $\begin{array}{l}\text { Análise dos acidentes de trabalho com } \\
\text { exposição a material biológico notificados } \\
\text { por profissionais da saúde }\end{array}$ & \multirow[b]{2}{*}{$\begin{array}{l}\text { Revista } \\
\text { Brasileira de } \\
\text { Medicina do } \\
\text { Trabalho }\end{array}$} & \multirow[b]{2}{*}{$\begin{array}{l}\text { Identificar o perfil epidemiológico dos profissionais } \\
\text { da saúde que sofreram acidentes de trabalho com } \\
\text { material biológico no ano de } 2017 \text { no município de } \\
\text { Canoas, Rio Grande do Sul }\end{array}$} & \multirow{2}{*}{$\begin{array}{l}\text { No ano de } 2017 \text { ocorreram } 121 \text { casos de acidente de trabalho } \\
\text { com exposição a material biológico. Houve predomínio de } \\
\text { acidentes em mulheres }(93,4 \%) \text {, da raça branca }(69,4 \%) \text {, na } \\
\text { faixa etária de } 20 \text { a } 30 \text { anos }(40,5 \%) \text {. Em relação ao tipo de } \\
\text { exposição, as percutâneas correspondem a } 76,8 \% \text { dos casos, } \\
\text { sendo o sangue o material orgânico mais prevalente }(90 \%) \text { e a } \\
\text { agulha com lúmen, o principal agente causador }(64,5 \%) \text {. A } \\
\text { luva foi o equipamento de proteção individual mais utilizado } \\
(75,2 \%) \text {, e os trabalhadores estavam vacinados em } 93,4 \% \text { dos } \\
\text { casos. }\end{array}$} \\
\hline & $\begin{array}{l}\text { Analysis of reported work accidents } \\
\text { involving healthcare workers and exposure } \\
\text { to biological materials }\end{array}$ & & & \\
\hline \multirow{2}{*}{$\begin{array}{l}\text { SOUSA AFL, et } \\
\text { al. (2016) }\end{array}$} & $\begin{array}{l}\text { Representações sociais da Enfermagem } \\
\text { sobre biossegurança: saúde ocupacional e } \\
\text { o cuidar prevencionista }\end{array}$ & \multirow{2}{*}{$\begin{array}{l}\text { Revista } \\
\text { Brasileira de } \\
\text { Enfermagem. }\end{array}$} & \multirow{2}{*}{$\begin{array}{l}\text { Apreender as representações sociais da } \\
\text { biossegurança por profissionais de Enfermagem na } \\
\text { Atenção Primária e analisar como elas se articulam } \\
\text { com a qualidade da assistência prestada. }\end{array}$} & \multirow{2}{*}{$\begin{array}{l}\text { Foram obtidas cinco classes: acidentes ocupacionais sofridos } \\
\text { pelos profissionais (19,66\%); exposição ocupacional a agentes } \\
\text { biológicos (14,04\%); gestão da biossegurança em Atenção } \\
\text { Primaria (12,92\%); importância do equipamento de proteção } \\
\text { individual }(26,97 \%) \text {, e biossegurança e controle de infecção } \\
(26,4 \%) \text {. }\end{array}$} \\
\hline & $\begin{array}{l}\text { Social representations of biosecurity in } \\
\text { nursing: occupational health and preventive } \\
\text { care }\end{array}$ & & & \\
\hline \multirow[b]{2}{*}{$\begin{array}{l}\text { TELES AS, et al. } \\
(2016)\end{array}$} & $\begin{array}{l}\text { Acidentes de trabalho com equipe de } \\
\text { enfermagem: uma revisão crítica }\end{array}$ & \multirow{2}{*}{$\begin{array}{l}\text { Revista de } \\
\text { Saúde Coletiva } \\
\text { da UEFS }\end{array}$} & \multirow{2}{*}{$\begin{array}{l}\text { Analisar, com base na literatura brasileira, os } \\
\text { acidentes entre os trabalhadores de enfermagem no } \\
\text { desenvolvimento de suas atividades laborais. }\end{array}$} & \multirow{2}{*}{$\begin{array}{l}\text { O principal acidente são os ferimentos com materiais } \\
\text { perfurocortantes ( } 21 \%) \text { envolvendo, principalmente, os } \\
\text { técnicos e auxiliares de enfermagem ( } 18 \%) \text {, sendo o local do } \\
\text { corpo mais atingido, as mãos }(7 \%) \text {. Os trabalhadores com } \\
\text { maior tempo de serviço se acidentaram mais }(4 \%) \text {, com } \\
\text { predomínio dos acidentes no turno matutino }(7 \%) \text {. }\end{array}$} \\
\hline & $\begin{array}{l}\text { Occupational accidents with nursing team: a } \\
\text { critical review }\end{array}$ & & & \\
\hline
\end{tabular}

Fonte: Rocha THL, et al., 2020.

REAS/EJCH | Vol.12(11) | e4035 | DOI: https://doi.org/10.25248/reas.e4035.2020Ｐágina 6 de 10 


\section{RESULTADOS E DISCUSSÃO}

A amostra desta revisão foi composta por 38 artigos, todavia apenas 21 foram selecionados, pelos critérios de inclusão estabelecidos, para a composição desse artigo. Constata-se que $14,28 \%(n=3)$ dos artigos foram publicados em 2016; 47,6\% ( $n=10)$ em 2017; 4,76\% ( $n=1) ; 28,57 \%(n=6)$ em 2019; e 4,76\% ( $n=1)$ em 2020.

A partir da leitura crítica dos artigos da amostra, foi possível perceber uma intrínseca relação entre fatores externos, como sexo, idade e profissão, e o uso incorreto de EPIs - causa frequente de acidentes com material biológico. Além disso, a ocorrência de tais incidentes pode ter agravos significativos e interfere no âmbito biopsicossocial do indivíduo, bem como afeta a economia do empregador e do Estado. Dessa forma, manifestaram-se três categorias para a discussão:

\section{Riscos de exposição ocupacional a agentes biológicos:}

O trabalhador, ao executar suas atividades, é submetido a riscos ocupacionais (RO). A promoção da Saúde do trabalhador exige o conhecimento sobre os determinantes da saúde que viabilizem mecanismos de intervenção para o seguro desenvolvimento das atividades. Segundo Fernandes MA, et al. (2017), existem aspectos subjetivos e coletivos destes riscos que estão diversas vezes associados à sobrecarga de trabalho, à negligência e à precariedade das condições para o exercício da profissão. Os riscos no ambiente de trabalho também estão relacionados à ignorância e/ou à falta de informação, destacando-se situações em que o profissional não suspeita da existência de tais vulnerabilidades. Os fungos, as bactérias e os vírus são agentes biológicos responsáveis por diversas infecções, crônicas e agudas.

A exposição do trabalhador a esses materiais biológicos, muitas vezes, ocorre por: a) exposição percutânea - quando o acidente envolve perfurocortante (PC) que provoque ruptura da barreira cutânea por incisão ou perfuração, b) exposição mucosa, c) exposição em pele não íntegra que apresente corte, arranhadura ou dermatite, d) arranhaduras ou mordeduras, quando envolvem presença de sangue (CUNHA NA, 2017). Concomitantemente a isso, o uso de Equipamentos de Proteção Individual (EPI), as condições clínicas, as medidas profiláticas, o grau de exposição, o tamanho da lesão são fatores que irão determinar o risco do acidente (SANDEIRO TL, et al., 2019).

Observa-se que as ocorrências de acidentes por inoculação percutânea através de material PC são consideradas de maior risco. Dentre os inúmeros patógenos que podem ser transmitidos, os vírus da AIDS (HIV), da Hepatite B (HBV) e da hepatite C (HCV) são os mais frequentes. Apesar da importância dessa problemática, a execução global das estratégias mostra-se falhas. Atualmente, existem 35 milhões de profissionais ligados à área da saúde, dentre estes mais de três milhões já tiveram algum tipo de exposição percutânea a vírus transmitidos por fluidos corporais.

Estudo revela que $70 \%$ da população mundial está coberta de alguma forma pela Atenção Primária à Saúde, entretanto, quando se trata de mecanismos de cuidado de saúde ocupacional na atenção primária, apenas 10 a $15 \%$ desses trabalhadores possuem acesso. Dessa maneira, percebe-se a importância da quimioprofilaxia logo após a exposição ao material biológico potencialmente infectado para minimizar os riscos de transmissão de doenças. (SOUSA AFL, et al., 2019).

Cabem salientar também os riscos inerentes à radioterapia e radiodiagnóstico, presentes em centros de terapia intensiva e unidades cirúrgicas. Em tais procedimentos, são utilizadas radiações ionizantes e não ionizantes, as quais podem acarretar alterações celulares - mutações e aumento da possibilidade de neoplasias - e queimaduras na pele e/ou olhos, respectivamente. Entretanto, tais agravos podem ser evitados, por exemplo, mediante a implantação de barreiras físicas, o uso de EPI específico e a capacitação profissional (BALTHAZAR MAP, et al., 2017).

\section{Grupos e procedimentos mais suscetíveis a falhas:}

Evidenciou-se que os trabalhadores da equipe de enfermagem apresentam maior vulnerabilidade a acidentes com materiais biológicos, haja vista a assistência direta e ininterrupta ao paciente, associada ao amplo manuseio de materiais perfurocortantes e ao grande número de procedimentos manuais realizados por esses profissionais. Sendo predominantes exposições a materiais biológicos em trabalhadores jovens, o que sugere inexperiência e inabilidade técnica, bem como a necessidade de melhor orientação quanto as medidas preventivas para tais acidentes (MIRANDA FMD, et al., 2017; SOARES RZ, et al., 2019). 
A respeito do gênero dos profissionais que sofreram exposições biológicas, há predomínio do sexo feminino, visto que as mulheres constituem a maioria dos profissionais de saúde, especialmente da equipe de enfermagem - reflexo de uma construção histórica, social e cultural nessa área (GOMES SCS, et al., 2019). É válido salientar que indivíduos sem responsabilidades familiares - solteiro divorciado ou viúvo apresentam um maior risco de exposição ocupacional, dada à diminuição dos níveis de proteção, como dos EPIs (LA-ROTTA EIG, et al., 2020). Outra condição facilitadora da ocorrência de acidentes ocupacionais com material biológico, é o crescente desgaste físico e mental do trabalhador, relacionado a sobrecarga de trabalho, a existência de mais de um vínculo empregatício e a horas extras na jornada laboral (FERREIRA LA, et al., 2017).

Constatou-se, ainda, maior frequência de acidentes no ambiente hospitalar, com ênfase para as unidades de internações, as quais são voltadas ao tratamento em acomodações adequadas para a prestação dos serviços, devido à realização de práticas mais complexas, ao maior contato com procedimentos invasivos e à admissão de pacientes com doenças infectocontagiosas. (ARANTES MC, et al., 2017; NEGRINHO NBS, et al., 2017). A maioria dos casos de exposição ocupacional com material biológico ocorreu por lesões percutâneas causadas pelo manuseio e descarte de materiais perfuro cortantes, principalmente agulhas com lúmen. Estima-se que, mundialmente, ocorram cerca de 926 mil acidentes percutâneos por ano entre profissionais da saúde (GOMES SCS, et al., 2019).

Ressalta-se a prevalência do sangue como material orgânico mais envolvido nos acidentes, sendo este um fluido potencialmente infectante e que pode conter elevada carga biológica, constituindo-se, portanto, de alto risco.

Em relação aos procedimentos referentes a acidentes percutâneos, destacam-se a administração de medicamentos e a punção de acesso vascular. Subsequentemente, realça-se a contaminação por contato direto com mucosas e/ou pele que apresenta rompimento da integridade (SARDEIRO TL, et al., 2019). Estudos sugerem que, embora os profissionais reconheçam o alto risco de contaminação presente em seu ambiente de trabalho, o elevado número de acidentes laborais com material biológico está ligado à baixa adesão ao uso de EPIs básicos, como luvas, óculos de proteção e máscaras, (CORDEIRO TMSC, et al., 2016).

A subutilização dos EPIs deve-se, essencialmente, a pouca abordagem dos princípios de biossegurança durante a prática clínica e de ensino, tal debilidade nos planos pedagógicos demonstra a limitada qualificação dos estudantes no que se refere à prática de segurança biológica. (BOEIRA ER, et al., 2019). Soma-se a isso, o descaso com o próprio equipamento por parte do empregador, o que vai de encontro, no Brasil, à norma regulamentadora seis (NR-06), segundo a qual instituição empregadora deve disponibilizar aos empregados EPIs adequados ao risco, conservados e com bom funcionamento (CARVALHO HEF, et al., 2019).

\section{Impactos socioeconômicos da falha no uso de EPIs:}

De um modo geral, percebe-se que os acidentes de trabalho são considerados, na atualidade, como um importante problema de saúde pública, tendo em vista os seus efeitos para as vítimas deste tipo de acidentes e para o sistema de saúde do país (CUNHA NA, 2017).

Identifica-se que a falha no uso de equipamentos de proteção acarreta prejuízos sociais e econômicos. As principais implicações são devido a acidentes de trabalho, os quais podem invalidar o profissional. A Lei $\mathrm{n}^{\circ}$ 8.213, de 24 de julho de 1991, afirma que acidente de trabalho é toda atividade que cause lesão a qual impeça a atuação permanente ou por tempo indeterminado ou que possa ocasionar a morte do profissional (BRASIL, 1991). Os estudos sobre a exposição sofrida por trabalhadores da área da saúde começaram após a transmissão ocupacional do vírus causador da síndrome da imunodeficiência humana (AIDS), alguns comportamentos que contribuíram para isso foram reencapar agulhas contaminadas e não usar proteção durante o manuseio de sangue e fluidos.

Vale ressaltar que esse tipo de infecção é acompanhado, além dos efeitos físicos da quimioprofilaxia e da própria patologia, por intensa carga emocional decorrente do receio da soroconversão, preocupação e alteração da dinâmica sociofamiliar (SOARES RZ, et al., 2016; CARVALHO DC, et al., 2018). Dependendo da natureza e da intensidade das exposições, pode haver danos à saúde dos profissionais expostos. As 
infecções ocorridas podem resultar em preocupantes consequências físicas - incapacitação profissional - e psicossociais - descontrole emocional - para a vítima, bem como para a corporação empregadora. Diante disso, destaca- se a ampliação dos níveis de absentismo, o que ocasiona prejuízos financeiros. Ademais, são necessários gastos com tratamentos e, muitas vezes, internações - o que amplia despesas institucionais e/ou estatais relativas à seguridade social.

É possível, ainda, que haja o aumento da resistência microbiana a antibióticos, uma vez que o profissional constantemente exposto a agentes biológicos tem maior necessidade de adotar medidas quimioprofiláticas, a fim de prevenir infecções ou evitar à evolução da doença, nos casos que já houve contaminação. Vale ressaltar também que há o risco de mortalidade pós-infecção decorrente da exposição biológica. Diante disso, é evidente que a ampliação de técnicas de segurança em procedimentos deve ser prioridade em ambientes de saúde, focalizando estratégias de promoção da saúde e de prevenção de tais incidentes (BOEIRA ER, et at., 2019; CARVALHO DC, et al., 2018).

Durante os atendimentos, os profissionais, muitas vezes sem a utilização correta dos EPIs e negligenciando a própria segurança, têm contato com pacientes sem diagnóstico de doenças que podem ser transmitidas por contato, principalmente fora das Unidades Básicas de Saúde (UBS), como exemplo durante visitas domiciliares, o que aumenta a suscetibilidade a contaminação biológica. (SOUSA AFL, et al., 2016).

A infecção hospitalar $(\mathrm{IH})$ é um dos principais tipos de contaminação a qual o profissional está exposto devido a maior quantidade de procedimentos invasivos. A Portaria № 2616/98 do MS conceitua Infecção Hospitalar como aquela adquirida após admissão do paciente e que se apresenta durante a internação ou após a alta. Esse tipo infecção é considerado problema de saúde pública, uma vez que a contaminação pode gerar gastos elevados para o Estado por necessitar, muitas vezes, de tratamentos de alto custo. (BATISTA JR, et al. 2017).

A Lei n 9431 de 1997 determina a existência do Controle de Infecção Hospitalar (PCIH) como órgão de autoridade e controle de infecções, pois esse programa aponta um conjunto de práticas que devem ser executadas minuciosamente visando diminuir a ocorrência e a intensidade das infecções no ambiente hospitalar (BRASIL, 1997). Além da necessidade de segurança durante a atuação do profissional, é indispensável que as técnicas de biossegurança sejam temática obrigatória na capacitação de novos profissionais, essas técnicas exigem constante revisão para evitar a proliferação de novos agentes patológicos.

A portaria atual que discorre sobre as ações e os serviços de saúde do Sistema Único de Saúde, no 5, de 28 de setembro de 2017, instituiu o Comitê Nacional de Promoção da Saúde do Trabalhador do Sistema Único de Saúde, com o objetivo de desenvolver estratégias de monitoramento dos riscos em ambientes de trabalho. É importante ressaltar que apenas o uso de EPIs não garante total segurança, sendo imprescindível a adesão a outras práticas de precaução-padrão como a higienização das mãos, descontaminação do ambiente, vacinação, entre outros (SOUSA AFL, et al., 2016).

\section{CONSIDERAÇÕES FINAIS}

Contempla-se, com a realização deste trabalho, uma melhor compreensão da ampla importância do uso dos Equipamentos de Proteção Individual, os quais auxiliam na promoção à biossegurança no ambiente de trabalho dos profissionais de saúde, também conduzem iniciativas de prevenção atreladas aos riscos de acidentes e associando-os aos impactos socioeconômicos que essas falhas possam trazer. Observou-se que os perigos estão relacionados, especialmente aos procedimentos de assistência ao paciente, à desinformação em relação aos riscos ocupacionais existentes no ambiente hospitalar por parte de certa parcela dos profissionais, e ao descuido associado a fatores externos que possam vir a interferir na passividade desses profissionais. Contudo, diversas são as medidas que podem e devem ser perfilhada para diminuir os acidentes ocupacionais, havendo a necessidade de uma centralização de empenhos e recursos para reconhecimento dos riscos no ambiente de trabalho, implantação de treinamentos, informativos espalhados pelo ambiente de trabalho, palestras abordando a conscientização de práticas seguras, aplicação do arcabouço presente nas Normas Regulamentadoras e fornecimento constante e uniforme dos dispositivos de segurança aos trabalhadores da área da saúde. 


\section{AGRADECIMENTO E FINANCIAMENTO}

Agradecemos a Faculdade de Ciências Humanas, Exatas e da Saúde do Piauí/ Instituto de Ensino Superior do Vale do Parnaíba por disponibilizar o local e os materiais para o desenvolvimento do projeto, e aos professores de Habilidades e Atitudes Médicas e profissionais da saúde, Dr. Augusto Beltrão e Dra. Renata Beltrão pelo incentivo da iniciação científica e a disponibilidade em auxiliar no desenvolvimento do estudo concedido. Não houve nenhum tipo de financiamento direcionado para a elaboração dessa revisão bibliográfica.

\section{REFERÊNCIAS}

1. ARANTES MC, et al. Acidentes de trabalho com material biológico em trabalhadores de serviço de saúde. Cogitare Enfermagem. 2017; 22(1): 01-08.

2. BALTHAZAR MAP, et al. Gestão dos riscos ocupacionais nos serviços hospitalares: uma análise reflexiva. Revista de Enfermagem UFPE. 2017; 11(9).

3. BATISTA JR, et al. Conhecimento da equipe de enfermagem perante os principais tipos de infecções hospitalares. Revista de Enfermagem UFPE. 2017; 11(12): 4946-5.

4. BOEIRA ER, et al. Controle de infecções e medidas de segurança do paciente abordados em projetos pedagógicos da enfermagem. Revista da Escola de Enfermagem USP. 2019; 53: e03420.

5. BRASIL. Lei $n^{\circ} 8.213$, de 24 de julho de 1991. Código Civil. Dispõe sobre os Planos de Benefícios da Previdência Social e dá outras providências.

6. BRASIL. Lei $n^{\circ}$ 9431, de 06 de janeiro de 1997, Código Civil. Dispõe sobre a obrigatoriedade da manutenção de programa de controle de infecções hospitalares pelos hospitais do país.

7. CARVALHO DC, et al. Acidentes de trabalho com material biológico na equipe de enfermagem de um hospital do Centro-Oeste brasileiro. Escola Anna Nery. 2018; (22)1.

8. CARVALHO HEF, et al. Visão dos Profissionais de Enfermagem Quanto aos Riscos Ocupacionais e Acidentes de Trabalho na Central de Material e Esterilização. Revista de Pesquisa: cuidado é fundamental. 2019; 11(5): $1161-1166$.

9. CORDEIRO TMSC, et al. Acidentes de trabalho com exposição à material biológico: Descrição dos casos na Bahia. Revista de Epidemiologia e Controle de Infecção. 2016; 6(2): 50-56.

10. CUNHA NA. Acidentes de trabalho com exposição a material biológico: análise epidemiológica e percepção das vítimas, em Uberlândia - MG. Instituto de geografia. Programa de pós-graduação em saúde ambiental e saúde do trabalhador. Uberlândia. 2017.

11. FERNANDES MA, et al. Utilização de equipamentos de proteção individual: interfaces com o conhecimento dos profissionais de saúde. Revista Prevenção de Infecção e Saúde. 2017; 3(1): 16-21.

12. GOMES SCS, CALDAS AJM. Incidência de acidentes de trabalho com exposição a material biológico em profissionais de saúde no Brasil, 2010-2016. Revista Brasileira de Medicina do Trabalho. 2019; 17(2): 188-200.

13. LA-ROTTA EIG, et al. Conhecimento e adesão como fatores associados a acidentes com agulhas contaminadas com material biológico: Brasil e Colômbia. Ciência \& Saúde Coletiva. 2020; 25(2): 715-727.

14. LIMA RJV, et al. Agentes biológicos e equipamentos de proteção individual e coletiva: conhecimento e utilização entre profissionais. Revista Prevenção de Infecção e Saúde. 2017; 3(1): 23-28.

15. MIRANDA FMD, et al. Perfil dos trabalhadores brasileiros vítimas de acidente de trabalho com fluidos biológicos. Revista Brasileira de Enfermagem. 2017; 70(5): 1061-8.

16. NEGRINHO NBS, et al. Fatores associados à exposição ocupacional com material biológico entre profissionais de enfermagem. Revista Brasileira de Enfermagem. 2017; 70(1): 126-31.

17. SALLES LL, ANJOS JM. Equipamentos de proteção individual no contexto laboral da enfermagem. Revista UNINGÁ. 2019; 56(S6): 134-147.

18. SARDEIRO TL, et al. Acidente de trabalho com material biológico: fatores associados ao abandono do acompanhamento clínico-laboratorial. Revista da Escola de Enfermagem da USP. 2019; 53: e03516.

19. SOARES RZ, et al. Análise dos acidentes de trabalho com exposição a material biológico notificados por profissionais da saúde. Revista Brasileira de Medicina do Trabalho. 2019; 17(2): $201-8$.

20. SOUSA AFL, et al. Representações sociais da enfermagem sobre biossegurança: saúde ocupacional e o cuidar prevencionista. Revista Brasileira de Enfermagem. 2016; 69(5): 810-7.

21. TELES AS, et al. Acidentes de trabalho com equipe de enfermagem: uma revisão crítica. Revista de Saúde Coletiva da UEFS, Feira de Santana, 2016; 6(1): 62-68. 